\title{
EVALUATION OF AGE GROUP OF 11,323 BREAST CANCER PATIENTS TREATED FROM JANUARY 2011 TO DECEMBER 2019 AT PEROLA BYINGTON HOSPITAL
}

Priscilla Maquinêz Veloso', Jorge Yoshinori Shida', Luiz Henrique Gebrim¹, André Mattar¹

${ }^{1}$ Hospital Pérola Byington-São Paulo (SP), Brazil.

Introduction: According to estimates from the Brazilian Department of Health, in 2021 we will see more than 65,000 cases of breast cancer in Brazil. The predominance of advanced cases in the Brazilian Unified Health System (SUS) stems from the long time for diagnosis and treatment of patients and, consequently, leads to a higher mortality rate. There is a lack of data on the age of our patients to establish an adequate coping strategy for the disease and thus reduce mortality in our country. The Department of Health recommends mammography from the age of 50. The Brazilian Society of Mastology (SBM), on the other hand, recommends exams starting at 40. Before that, only for groups at risk. In 2018, there were 2,1 million new cases, equivalent to $11.6 \%$ of all estimated cancers. This value corresponds to an estimated risk of 55.2/100 thousand. The highest expected incidence rates were in Australia and New Zealand, in Northern European countries and in Western Europe. Regardless of the country's socioeconomic situation, the incidence of this cancer ranks among the top positions for female malignancies. On the other hand, there has been a decline in the trend of incidence rates in some developed countries, partly linked to the decrease in hormone replacement therapy in postmenopausal women. Objectives: This paper aims to describe treatment of breast cancer according the age group of 11,323 women by SUS at Pérola Byington Hospital from January 2011 to December 2019. Methods: A hospital-based observational crosssectional study was carried out, in which the eligible population consisted of 11,323 patients with breast cancer treated by SUS at Pérola Byington Hospital whose data was registered in the data collection system of that hospital. Women under the age of 20 years up to over 80 years were selected. Results: A predominance of the diagnosis was observed in women aged 50 to 59 years (27.91\%), followed by patients aged 40 to 49 years $(23.90 \%)$ and by patients aged 60 to 69 years $(22.26 \%)$. Women under the age of 20 were diagnosed in $0.06 \%$ of cases and over 80 years of age in $4.75 \%$. Conclusions: The diagnosis of breast cancer in women under 40 years of age is rare, representing around $10 \%$ of all registered cases. But, when it occurs in this age group, the disease tends to be more aggressive, raising a question of from what age the screening test should be performed. 